Georgian Mathematical Journal

1(1994), No. 2, 213-227

\title{
ON INTEGRAL BERNSTEIN OPERATORS IN SOME CLASSES OF MEASURABLE BIVARIATE FUNCTIONS
}

\author{
ROMAN TABERSKI
}

\begin{abstract}
The two main theorems are cencerned with the approximation of (complex-valued) functions on the real plane by sums of Bernstein pseudoentire functions. They are formulated and proved in Section 4, after prior determination of the suitable integral operators. Analogous results for pseudopolynomial approximations were obtained by Brudnyi, Gonska, and Jetter ([2],[3]).
\end{abstract}

1. Preliminaries. Let $L_{l o c}(R)$ [resp. $A C_{l o c}(R)$ ] be the set of all univariate (complex-valued) functions Lebesgue-integrable (absolutely continuous) on every compact subinterval of $R:=(-\infty, \infty)$. Denote by $L_{l o c}^{p}\left(R^{2}\right)$, $1 \leq p \leq \infty$, the set of all measurable bivariate (complex-valued) functions Lebesgue-integrable with pth power (essentially bounded when $p=\infty$ ) on every finite two-dimensional integral lying on the plane $R^{2}=R \times R$; write $L_{l o c}\left(R^{2}\right)$ instead of $L_{l o c}^{1}\left(R^{2}\right)$. Denote by $C(R)$ [resp. $\left.C\left(R^{2}\right)\right]$ the set of all (complex-valued) functions continuous on $R\left[R^{2}\right]$.

Given any bivariate (complex-valued) function $f \equiv f(\cdot, \cdot)$ measurable on $R^{2}$, the quantity

$$
\|f\|_{p}:= \begin{cases}\left(\iint_{R^{2}}|f(u, v)|^{p} d u d v\right)^{1 / p} & \text { if } 1 \leq p<\infty \\ \operatorname{ess}_{\sup } \sup _{(u, v) \in R^{2}}|f(u, v)| & \text { if } p=\infty\end{cases}
$$

is finite or infinite. In the case $\|f\|_{p}<\infty$ the function $f$ is said to be of class $L^{p} \equiv L^{p}\left(R^{2}\right)$; in symbols, $f(\cdot, \cdot) \in L^{p}$. The notations $f(\cdot, \cdot) \in L_{l o c}^{p}\left(R^{2}\right)$, $g(\cdot, v) \in L_{l o c}(R)$, etc. have a similar meaning.

Let $f \equiv f(\cdot, \cdot)$ be a (complex-valued) function defined on $R^{2}$ and let $k, l \in N_{0} \equiv\{0,1,2, \ldots\}$. Determine the partial differences of $f$ at a point

1991 Mathematics Subject Classification. 41A25, 41A35.

* Research supported by KBN grant 210799101. 
$(x, y) \in R^{2}$, i.e.

$$
\begin{aligned}
{ }^{1} \Delta_{\lambda}^{k} f(x, y) & :=\sum_{\mu=0}^{k}(-1)^{k-\mu}\left(\begin{array}{l}
k \\
\mu
\end{array}\right) f(x+\mu \lambda, y), \\
{ }^{1} \Delta_{\eta}^{l} f(x, y) & :=\sum_{\nu=0}^{k}(-1)^{l-\nu}\left(\begin{array}{l}
l \\
\nu
\end{array}\right) f(x, y+\nu \eta),
\end{aligned}
$$

with the real increments $\lambda, \eta$. Introduce also the mixed difference $\Delta_{\lambda, \eta}^{k, l}:=$ ${ }^{1} \Delta_{\lambda}^{k}\left({ }^{2} \Delta_{\eta}^{l} f(x, y)\right)$.

It is easy to see that

$$
\Delta_{\lambda, \eta}^{k, l}=\sum_{\mu=0}^{k} \sum_{\nu=0}^{l}(-1)^{k+l-\mu-\nu}\left(\begin{array}{l}
k \\
\mu
\end{array}\right)\left(\begin{array}{l}
l \\
\nu
\end{array}\right) f(x+\mu \lambda, y+\nu \eta) .
$$

In particular, $\Delta_{\lambda, \eta}^{0,0} f(x, y)=f(x, y)$ and

$$
\Delta_{\lambda, \eta}^{1,1} f(x, y)=f(x+\lambda, y+\eta)-f(x+\lambda, y)-f(x, y+\eta)+f(x, y) .
$$

Further, $\Delta_{\lambda, \eta}^{1,1}\left(\Delta_{\lambda, \eta}^{k, l} f(x, y)\right)=\Delta_{\lambda, \eta}^{k+1, l+1} f(x, y)$.

The first (weak) derivative of $f$ at $(x, y)$ is given by

$$
f^{(1)}(x, y):=\lim _{\lambda \rightarrow 0} \frac{1}{\lambda^{2}} \Delta_{\lambda, \lambda}^{1,1} f(x, y)
$$

whenever the right-hand side exists. The (weak) derivatives of $f$ of higher orders are defined successively:

$$
f^{(j)}(x, y):=\left(f^{(j-1)}\right)^{(1)}(x, y) \text { for } j=2,3, \ldots
$$

Moreover, by convention, $f^{(0)}(x, y) \equiv f(x, y)$ and

$$
f^{(m, n)}(x, y) \equiv \frac{\partial^{m}}{\partial x^{m}}\left(\frac{\partial^{n}}{\partial y^{n}} f(x, y)\right) \text { for } m, n \in N_{0} .
$$

Considering any (complex-valued) function $f$ measurable on $R^{2}$, one can define its mixed $L^{p}$-modulus of smoothness:

$$
\omega_{k, l}\left(\delta_{1}, \delta_{2} ; f\right)_{p}:=\sup \left\{\left\|\Delta_{\lambda, \eta}^{k, l} f\right\|_{p}: 0 \leq \lambda \leq \delta_{1}, 0 \leq \eta \leq \delta_{2}\right\} .
$$

This quantity, with fixed $p \geq 1$ and $k, l \in N_{0}$, may be finite or infinite for positive numbers $\delta_{1}, \delta_{2}$. If there exist three non-negative numbers $M, \alpha, \beta$ such that $\omega_{k, l}\left(\delta_{1}, \delta_{2} ; f\right)_{p} \leq M \delta_{1}^{\alpha} \delta_{2}^{\beta}$ for all $\delta_{1}, \delta_{2} \in(0,1]$, we say that $f$ belongs to the Hölder class $H_{\alpha, \beta, p}^{(k, l)}$. More generally, if

$$
\|f\|_{\varphi, \psi, p}^{(k, l)} \equiv \sup \left\{\frac{1}{\varphi(\lambda) \psi(\eta)}\left\|\Delta_{\lambda, \eta}^{k, l} f\right\|_{p}: 0<\lambda, \eta \leq 1\right\}<\infty,
$$


where $\varphi, \psi$ mean positive non-decreasing functions on $(0,1]$ and $\varphi(1)=$ $\psi(1)=1$, the function $f$ is said to be of class $H_{\varphi, \psi, p}^{(k, l)}$. In the case $\varphi(\delta)=\delta^{\alpha}$, $\psi(\delta)=\delta^{\beta}$ for $\delta \in(0,1]$, the left-hand side of identity (2) will be denoted by $\|f\|_{\alpha, \beta, p}^{(k, l)}$.

Denote by $E_{\sigma}$ [resp. $E_{\sigma, \tau}$ ] the set of all univariate (bivariate) entire functions of exponential type of order $\sigma[(\sigma, \tau)]$ at most. Clearly, if $F(\cdot, \cdot) \in$ $E_{\sigma, \tau}(\sigma, \tau \geq 0)$, then $F(\cdot, v) \in E_{\sigma}$ and $F(u, \cdot) \in E_{\tau}$ for all $u, v \in R$. Moreover, $F(\cdot, \cdot) \in C\left(R^{2}\right)$. In the case where $\Phi(\cdot, v) \in E_{\sigma}\left(\right.$ resp. $\left.\Psi(u, \cdot) \in E_{\tau}\right)$ for almost every $v \in R[u \in R], \Phi(z, \cdot) \in L_{l o c}(R)\left[\Psi(\cdot, z) \in L_{l o c}\left(R^{2}\right)\right]$ for every complex number $z$, and $\Phi(\cdot, \cdot) \in L_{l o c}\left(R^{2}\right)\left[\Psi(\cdot, \cdot) \in L_{l o c}\left(R^{2}\right)\right]$, we call $\Phi[\Psi]$ a pseudoentire function of class $W_{\sigma}^{1}\left[W_{\tau}^{2}\right]$.

The aim of this paper is to present the Jackson type theorems, in $L^{p_{-}}$ norms (1) and seminorms (2), for some (complex-valued) functions defined and measurable on $R^{2}$. We begin with auxiliary results about the mixed differences and Bernstein's singular integrals used in our approximation problems.

2. Estimates for the mixed differences and moduli of smoothness. Consider a (complex-valued) function $f \equiv f(\cdot, \cdot)$ defined and measurable on the plane $R^{2}$. Denote by $k, l$ two non-negative integers. Take an arbitrary $p$ satisfying the condition: $1 \leq p \leq \infty$.

Proposition 1. If $\lambda, \eta \in R$ and $n \in N \equiv\{1,2,3, \ldots\}$, then

$$
\left\|\Delta_{m \lambda, n \eta}^{k, l} f\right\|_{p} \leq m^{k} n^{l}\left\|\Delta_{\lambda, \eta}^{k, l} f\right\|_{p}
$$

Proof. Given arbitrary $x, y \in R$, let $g(x, y):={ }^{2} \Delta_{n \eta}^{l} f(x, y)$.

By identity (5) of [5], p.116,

$$
g(x, y)=\sum_{\nu_{1}=0}^{n-1} \cdots \sum_{\nu_{l}=0}^{n-1} 2 \Delta_{\eta}^{l} f\left(x, y+\nu_{1} \eta+\cdots+\nu_{i} \eta\right)
$$

and

$$
{ }^{1} \Delta_{m \lambda}^{k} g(x, y)=\sum_{\mu_{1}=0}^{m-1} \cdots \sum_{\mu_{k}=0}^{m-1}{ }^{1} \Delta_{\lambda}^{k} g\left(x+\mu_{1} \lambda+\cdots+\mu_{k} \lambda, y\right) .
$$

Hence

$$
\begin{gathered}
{ }^{1} \Delta_{m \lambda, n \eta}^{k, l} f(x, y)=\sum_{\mu_{1}=0}^{m-1} \cdots \sum_{\mu_{k}=0}^{m-1} \sum_{\nu_{1}=0}^{n-1} \cdots \\
\cdots \sum_{\nu_{l}=0}^{n-1} \Delta_{\lambda, \eta}^{k, l} f\left(x+\mu_{1} \lambda+\cdots+\mu_{k} \lambda, y+\nu_{1} \eta+\cdots+\nu_{i} \eta\right) .
\end{gathered}
$$

Applying Minkowski's inequality, we get at once estimate (3). 
From (3) it follows that

$$
\omega_{k, l}\left(m \delta_{1}, n \delta_{2} ; f\right)_{p} \leq m^{k} n^{l} \omega_{k, l}\left(\delta_{1}, \delta_{2} ; f\right)_{p} \quad(m, n \in N)
$$

for all non-negative numbers $\delta_{1}, \delta_{2}$, whence, in case $a, b \geq 0$

$$
\omega_{k, l}\left(a \delta_{1}, b \delta_{2} ; f\right)_{p} \leq([a]+1)^{k}([b]+1)^{l} \omega_{k, l}\left(\delta_{1}, \delta_{2} ; f\right)_{p} .
$$

Clearly, the estimates (3)-(5) are useful only with the finite right-hand sides.

Proposition 2. Let $f$ have the partial derivatives

$$
f^{(0, n)}(u, \cdot), \ldots, f^{(m-1, n)}(u, \cdot) \in L_{l o c}(R)(m, n \in N)
$$

for every $u \in R$, and let $f^{(m-1, n)}(\cdot, v) \in A C_{l o c}(R)$ for almost every $v \in R$. Further, given any $c>0$, suppose the existence of positive number $M_{c}$ such that ess $\sup _{-c \leq u \leq c}\left|f^{(m, n)}(u, v)\right| \leq M_{c}$ for almost every $v \in[-c, c]$. Then, in the case $k \geq m$ and $\lambda, \eta \in R$,

$$
\left\|\Delta_{\lambda, \eta}^{k, l} f\right\|_{p} \leq|\lambda|^{m}|\eta|^{n}\left\|\Delta_{\lambda, \eta}^{k-m, l-n} f^{(m, n)}\right\|_{p} .
$$

Proof. By our assumption, the (Lebesgue) integrals

$$
\begin{gathered}
\int_{0}^{\eta} \cdots \int_{0}^{\eta} f^{(j, n)}\left(u, w+t_{1}+\cdots+t_{n}\right) d t_{1} \cdots d t_{n} \equiv F_{j}(u, w) \\
(j=0,1, \ldots, m)
\end{gathered}
$$

exist for all $u, w \in R$ if $j<m$ and for almost every $u$ and every $w \in R$ if $j=m$.

Given $u, w \in R$, one has

$$
\begin{gathered}
\left|\frac{1}{h} \int_{0}^{h} f^{(m, n)}\left(u+s, w+t_{1}+\cdots+t_{n}\right) d s\right| \leq M_{c} \\
\text { uniformly in } h \in[-1,1]
\end{gathered}
$$

$(h \neq 0)$ for almost every point $\left(t_{1}, \cdots, t_{n}\right)$ of the $n$-dimensional interval $[-|\eta|,|\eta|]^{n}$ whenever $c \geq \max (|u|+1,|w|+n|\eta|)$. Moreover,

$$
\begin{gathered}
\lim _{h \rightarrow 0} \frac{1}{h} \int_{0}^{h} f^{(m, n)}\left(u+s, w+t_{1}+\cdots+t_{n}\right) d s= \\
=f^{(m, n)}\left(u, w+t_{1}+\cdots+t_{n}\right)
\end{gathered}
$$

for almost every $u$. Hence

$$
\begin{gathered}
\lim _{h \rightarrow 0} \frac{1}{h}\left\{F_{m-1}(u+h, w)-F_{m-1}(u, w)\right\}= \\
=\int_{0}^{\eta} \cdots \int_{0}^{\eta} f^{(m, n)}\left(u, w+t_{1}+\cdots+t_{n}\right) d t_{1} \cdots d t_{n}
\end{gathered}
$$


i.e., $F_{m-1}^{(1,0)}(u, w)=F_{m}(u, w)$ for a.e. $u \in R$ and every $w \in R$, by the Lebesgue dominated convergence theorem.

Next, when $m \geq 2,(u, w) \in R^{2}$ and $0<|h| \leq 1$,

$$
\begin{gathered}
\frac{1}{h}\left\{F_{m-2}(u+h, w)-F_{m-2}(u, w)\right\}= \\
=\int_{0}^{\eta} \cdots \int_{0}^{\eta}\left\{\frac{1}{h} \int_{0}^{h} f^{(m-1, n)}\left(u+s, w+t_{1}+\cdots+t_{n}\right) d s\right\} d t_{1} \cdots d t_{n}, \\
\left|\frac{1}{h} f^{(m-1, n)}\left(u+s, w+t_{1}+\cdots+t_{n}\right) d s\right|= \\
=\mid \frac{1}{h}\left\{\int_{0}^{s} f^{(m, n)}\left(u+z, w+t_{1}+\cdots+t_{n}\right) d z+\right. \\
\left.+f^{(m-1, n)}\left(u, w+t_{1}+\cdots+t_{n}\right)\right\} d s \mid \leq \\
\leq\left|\frac{1}{h}\right| f^{(m, n)}\left(u+z, w+t_{1}+\cdots+t_{n}\right)|d z|+ \\
+\left|f^{(m-1, n)}\left(u, w+t_{1}+\cdots+t_{n}\right)\right|
\end{gathered}
$$

and

$$
\begin{gathered}
\lim _{h \rightarrow 0} \frac{1}{h} \int_{0}^{h} f^{(m-1, n)}\left(u+s, w+t_{1}+\cdots+t_{n}\right) d s= \\
=f^{(m-1, n)}\left(u, w+t_{1}+\cdots+t_{n}\right)
\end{gathered}
$$

for almost all $\left(t_{1}, \ldots, t_{n}\right) \in[-|\eta|,|\eta|]^{n}$. Therefore, as previously, $F_{m-2}^{(1,0)}(u, w)=F_{m-1}(u, w)$ for all $(u, w) \in R^{2}$ Analogously, when $m \geq 3$, $F_{m-3}^{(1,0)}(u, w)=F_{m-2}(u, w)$ for all $(u, w) \in R^{2}$, etc. Consequently, if $m \geq$ $1, F_{0}^{(m-1,0)}(u, w)=F_{m-1}(u, w)$ for all $(u, w) \in R^{2}$, and $F_{0}^{(m, 0)}(u, w)=$ $F_{m}(u, w)$ for a.e. $u \in R$ and every $w \in R$

Further, in the case $-c \leq a \leq b \leq c$ and $|w|+n|\eta| \leq c$,

$$
\begin{gathered}
\left|F_{m-1}(b, w)-F_{m-1}(a, w)\right|= \\
=\mid \int_{0}^{\eta} \cdots \int_{0}^{\eta}\left\{\int_{a}^{b} f^{(m, n)}\left(s, w+t_{1}+\cdots+t_{n}\right) d s\right\} d t_{1} \cdots d t_{n} \leq \\
\leq M_{c}(b-a)|\eta|^{n} .
\end{gathered}
$$

Hence $F_{m-1}(\cdot, w) \in A C_{l o c}(R)$ for every $w \in R$. 
By identity (4) of [5], p.116,

$$
\begin{gathered}
\Delta_{\lambda, \eta}^{m, n} f(x, y)={ }^{1} \Delta_{\lambda}^{m}\left({ }^{2} \Delta_{\eta}^{n} f(x, y)\right)={ }^{1} \Delta_{\lambda}^{m} F_{0}(x, y)= \\
=\int_{0}^{\lambda} \cdots \int_{0}^{\lambda} F_{0}^{(m, 0)}\left(x+s_{1}+\cdots+s_{m}, y\right) d s_{1} \cdots d s_{m}= \\
=\int_{0}^{\lambda} \cdots \int_{0}^{\lambda} F_{m}\left(x+s_{1}+\cdots+s_{m}, y\right) d s_{1} \cdots d s_{m}
\end{gathered}
$$

for arbitrary $x, y, \lambda, \eta \in R$. Thus

$$
\begin{gathered}
\Delta_{\lambda, \eta}^{m, n} f(x, y)=\int_{0}^{\lambda} \cdots \int_{0}^{\lambda}\left\{\int_{0}^{\eta} \ldots\right. \\
\left.\cdots \int_{0}^{\eta} f^{(m, n)}\left(x+s_{1}+\cdots+s_{m}, y+t_{1}+\cdots+t_{n}\right) d t_{1} \ldots d t_{n}\right\} d s_{1} \ldots d s_{m} .
\end{gathered}
$$

Observing that

$$
\begin{gathered}
\Delta_{\lambda, \eta}^{k, l} f(x, y)=\Delta_{\lambda, \eta}^{k-m, l-n}\left(\Delta_{\lambda, \eta}^{m, n} f(x, y)\right)= \\
=\int_{0}^{\lambda} \cdots \int_{0}^{\lambda}\left\{\int_{0}^{\eta} \cdots \int_{0}^{\eta} \Delta_{\lambda, \eta}^{k-m, l-n} \times\right. \\
\left.\times f^{(m, n)}\left(x+s_{1}+\cdots+s_{m}, y+t_{1}+\cdots+t_{n}\right) d t_{1} \cdots d t_{n}\right\} d s_{1} \cdots d s_{m}
\end{gathered}
$$

and applying the generalized Minkowski's inequality, we obtain (6).

Estimate (6) immediately implies

$$
\omega_{k, l}\left(\delta_{1}, \delta_{2} ; f\right)_{p} \leq \delta_{1}^{m} \delta_{2}^{n} \omega_{k-m, l-n}\left(\delta_{1}, \delta_{2} ; f^{(m, n)}\right)_{p} \text { for } \delta_{1}, \delta_{2} \geq 0 .
$$

Proposition 3. Let $\left(f_{0}, f_{1}, \ldots, f_{\rho}\right)$ be a system of (complex-valued) functions of two real variables, such that $f_{\rho} \in L_{l o c}\left(R^{2}\right)(\rho \in N)$ and, for $j=1, \ldots, \rho$,

$$
\begin{gathered}
f_{\rho-j}(x, y)=f_{\rho-j}(x, 0)+f_{\rho-j}(0, y)-f_{\rho-j}(0,0)+ \\
+\int_{0}^{x} \int_{0}^{y} f_{\rho-j+1}(u, v) d u d v \quad \text { if } x, y \neq 0,
\end{gathered}
$$

$f_{\rho-j}(x, 0), f_{\rho-j}(0, y)$ are defined for all real $x, y$ and, when $j<\rho, f_{\rho-j}(\cdot, 0)$, $f_{\rho-j}(0, \cdot) \in L_{l o c}(R)$. Suppose that the integer $k$ is greater than or equal to $\rho$. Then, for all $\lambda, \eta \in R$

$$
\left\|\Delta_{\lambda, \eta}^{k, k} f_{0}\right\|_{p} \leq|\lambda \eta|^{\rho}\left\|\Delta_{\lambda, \eta}^{k-\rho, k-\rho} f_{\rho}\right\|_{p} .
$$


Proof. Given arbitrary $x, y, \lambda, \eta \in R$, we have

$$
\begin{gathered}
\Delta_{\lambda, \eta}^{1,1} f_{0}(x, y)=\int_{x}^{x+y} \int_{y}^{y+\eta} f_{1}(s, t) d s d t= \\
=\int_{0}^{\lambda} \int_{0}^{\eta} f_{1}\left(x+s_{1}, y+t_{1}\right) d s_{1} d t_{1} \\
\Delta_{\lambda, \eta}^{2,2} f_{0}(x, y)=\int_{0}^{\lambda} \int_{0}^{\eta} \Delta_{\lambda, \eta}^{1,1} f_{1}\left(x+s_{1}, y+t_{1}\right) d s_{1} d t_{1}= \\
=\int_{0}^{\lambda} \int_{0}^{\eta}\left\{\int_{0}^{\lambda} \int_{0}^{\eta} f_{2}\left(x+s_{1}+s_{2}, y+t_{1}+t_{2}\right) d s_{2} d t_{2}\right\} d s_{1} d t_{1}, \text { etc. }
\end{gathered}
$$

Therefore

$$
\begin{gathered}
\Delta_{\lambda, \eta}^{k, k} f_{0}(x, y)=\Delta_{\lambda, \eta}^{k-\rho, k-\rho}\left(\Delta_{\lambda, \eta}^{\rho, \rho} f_{0}(x, y)\right)= \\
=\int_{0}^{\lambda} \cdots \int_{0}^{\lambda} \int_{0}^{\eta} \cdots \int_{0}^{\eta} \Delta_{\lambda, \eta}^{k-\rho, k-\rho} \times \\
\times f_{\rho}\left(x+s_{1}+\cdots+s_{\rho}, y+t_{1}+\cdots+t_{\rho}\right) d s_{1} \cdots d s_{\rho} d t_{1} \cdots d t_{\rho} .
\end{gathered}
$$

This immediately implies estimate (8).

From (8) it follows that

$$
\omega_{k, k}\left(\delta_{1}, \delta_{2} ; f_{0}\right)_{p} \leq\left(\delta_{1} \delta_{2}\right)^{\rho} \omega_{k-\rho, k-\rho}\left(\delta_{1}, \delta_{2} ; f_{\rho}\right)_{p}
$$

for all non-negative numbers $\delta_{1}, \delta_{2}$.

3. Basic properties of the Bernstein singular integrals. Consider the entire functions $g_{\sigma}, G_{\sigma, k}$ of exponential type of positive order $\sigma$, with positive integer parameters $r, k$ given by

$$
g_{\sigma}(z):=\left(\frac{1}{z} \sin \frac{\sigma z}{2 r}\right)^{2 r}, \quad G_{\sigma, k}(\zeta):=\sum_{\mu=1}^{k}(-1)^{\mu} \frac{1}{\mu}\left(\begin{array}{c}
k \\
\mu
\end{array}\right) g_{\sigma}\left(\frac{\zeta}{\mu}\right)
$$

Write

$$
\gamma_{\sigma}:=\int_{R} g_{\sigma}(t) d t=2\left(\frac{\sigma}{2 r}\right)^{2 r-1} \int_{0}^{\infty}\left(\frac{\sin v}{v}\right)^{2 r} d v .
$$

Suppose that $f \equiv f(\cdot, \cdot)$ is a (complex-valued) function defined and measurable on $R^{2}$, such that

$$
I(f) \equiv \iint_{R^{2}} \frac{|f(u, v)|}{\left(1+u^{2 r}\right)\left(1+v^{2 r}\right)} d u d v<\infty .
$$


Take arbitrary $\tau>0, l \in N$ and complex numbers $z_{1}=x_{1}+i y_{1}, z_{2}=$ $x_{2}+i y_{2}\left(x_{m}, y_{m} \in R\right)$. Introduce the singular integrals

$$
\begin{gathered}
J_{\sigma, \tau}[f]\left(z_{1}, z_{2}\right):=\left(\gamma_{\sigma} \gamma_{\tau}\right)^{-1} \iint_{R^{2}} f(u, v) G_{\sigma, k}\left(z_{1}-u\right) G_{\tau, l}\left(z_{2}-v\right) d u d v \\
\left.J_{\sigma}^{1}[f]\left(z_{1}, v\right):=\gamma_{\sigma}^{-1} \int_{R} f(u, v) G_{\sigma, k}\left(z_{1}-u\right)\right) d u \quad(v \in R), \\
J_{\tau}^{2}[f]\left(u, z_{2}\right):=\gamma_{\tau}^{-1} \int_{R} f(u, v) G_{\tau, l}\left(z_{2}-v\right) d v \quad(u \in R),
\end{gathered}
$$

which are due to S.Bernstein ([1], pp.421-432).

The double integral (11) exists in the Lebesgue sense for all complex $z_{1}, z_{2}$; the single (Lebesgue) integrals (12) and (13) exist for every complex $z_{1}$ [resp. $z_{2}$ ] and almost every real $v$ [resp. $u$ ]. More precise assertions will be presented below.

Proposition 4. The relation

$$
J_{\sigma, \tau}[f]\left(z_{1}, z_{2}\right)=O\left(\left(1+x_{1}^{2 r}\right)\left(1+x_{2}^{2 r}\right) e^{\sigma\left|y_{1}\right|+\tau\left|y_{2}\right|} I(f)\right)
$$

holds uniformly in $x_{1}, y_{1}, x_{2}, y_{2} \in R$; moreover, $J_{\sigma, \tau}[f]$ coincides with some bivariate entire function.

Proof. Putting

$$
S\left(z_{1}, z_{2}\right):=\iint_{R^{2}} f(u, v) g_{\sigma}\left(z_{1}-u\right) g_{\tau}\left(z_{2}-v\right) d u d v,
$$

we can, formally, write

$$
\begin{gathered}
S\left(z_{1}, z_{2}\right)=\left(\int_{x_{1}-1}^{x_{1}+1} \int_{x_{2}-1}^{x_{2}+1}+\int_{x_{1}+1}^{\infty} \int_{x_{2}+1}^{\infty}+\right. \\
\left.+\int_{x_{1}+1}^{\infty} \int_{x_{2}-1}^{x_{2}+1}+\cdots\right) f(u, v) g_{\sigma}\left(z_{1}-u\right) g_{\tau}\left(z_{2}-v\right) d u d v= \\
=T_{0}\left(z_{1}, z_{2}\right)+T_{1}\left(z_{1}, z_{2}\right)+T_{2}\left(z_{1}, z_{2}\right)+\cdots .
\end{gathered}
$$

In view of $\left.\left.(10), f \in L_{l o c}\right) R^{2}\right)$. Hence the term $T_{0}\left(z_{1}, z_{2}\right)$ exists because $g_{\sigma} \in E_{\sigma}, g_{\tau} \in E_{\tau}$. Furthermore,

$$
\begin{gathered}
\left|T_{0}\left(z_{1}, z_{2}\right)\right| \leq \int_{x_{1}-1}^{x_{1}+1} \int_{x_{2}-1}^{x_{2}+1}|f(u, v)|\left(\frac{\sigma}{2 r}\right)^{2 r} e^{\sigma\left|y_{1}\right|}\left(\frac{\tau}{2 r}\right)^{2 r} e^{\tau\left|y_{2}\right|} d u d v \leq \\
\leq\left(\frac{\sigma \tau}{4 r^{2}}\right)^{2 r} e^{\sigma\left|y_{1}\right|+\tau\left|y_{2}\right|} \int_{x_{1}-1}^{x_{1}+1} \int_{x_{2}-1}^{x_{2}+1} \frac{|f(u, v)|}{\left(1+u^{2 r}\right)\left(1+v^{2 r}\right)} d u d v . \\
\cdot\left\{1+\left(\left|x_{1}\right|+1\right)^{2 r}\right\}\left\{1+\left(\left|x_{2}\right|+1\right)^{2 r}\right\} \leq \\
\leq 4\left(\frac{\sigma \tau}{4 r^{2}}\right)^{2 r} e^{\sigma\left|y_{1}\right|+\tau\left|y_{2}\right|} I(f)\left(\left|x_{1}\right|+1\right)^{2 r}\left(\left|x_{2}\right|+1\right)^{2 r} .
\end{gathered}
$$


Next

$$
\begin{gathered}
\left|T_{1}\left(z_{1}, z_{2}\right)\right| \leq \int_{x_{1}+1}^{\infty} \int_{x_{2}+1}^{\infty}|f(u, v)| e^{\sigma\left|y_{1}\right|}\left(x_{1}-u\right)^{-2 r} e^{\tau\left|y_{2}\right|}\left(x_{2}-v\right)^{-2 r} d u d v \leq \\
\leq e^{\sigma\left|y_{1}\right|+\tau\left|y_{2}\right|} \int_{x_{1}+1}^{\infty} \int_{x_{2}+1}^{\infty} \frac{|f(u, v)|}{\left(1+u^{2 r}\right)\left(1+v^{2 r}\right)} d u d v . \\
\cdot\left\{1+\left(\left|x_{1}\right|+1\right)^{2 r}\right\}\left\{1+\left(\left|x_{2}\right|+1\right)^{2 r}\right\} \leq \\
\leq 4 e^{\sigma\left|y_{1}\right|+\tau\left|y_{2}\right|} I(f)\left(\left|x_{1}\right|+1\right)^{2 r}\left(\left|x_{2}\right|+1\right)^{2 r}, \\
\left|T_{2}\left(z_{1}, z_{2}\right)\right| \leq \int_{x_{1}+1}^{\infty} \int_{x_{2}-1}^{\infty}|f(u, v)| e^{\sigma\left|y_{1}\right|}\left(x_{1}-u\right)^{-2 r}\left(\frac{\tau}{2 r}\right)^{2 r} e^{\tau\left|y_{2}\right|} d u d v \leq \\
\leq 4\left(\frac{\tau}{2 r}\right)^{2 r} e^{\sigma\left|y_{1}\right|+\tau\left|y_{2}\right|} I(f)\left(\left|x_{1}\right|+1\right)^{2 r}\left(\left|x_{2}\right|+1\right)^{2 r}, \text { etc. }
\end{gathered}
$$

Thus

$$
S\left(z_{1}, z_{2}\right)=O\left(\left(1+x_{1}^{2 r}\right)\left(1+x_{2}^{2 r}\right) e^{\sigma\left|y_{1}\right|+\tau\left|y_{2}\right|} I(f)\right)
$$

uniformly in $x_{1}, y_{1}, x_{2}, y_{2} \in R$.

Evidently, the left-hand side of (15) can be replaced by

$$
S_{\mu, \nu}\left(z_{1}, z_{2}\right):=\iint_{R^{2}} f(u, v) g_{\sigma}\left(\frac{z_{1}-u}{\mu}\right) g_{\tau}\left(\frac{z_{2}-v}{\nu}\right) d u d v(\mu, \nu \in N) .
$$

Further,

$$
J_{\sigma, \tau}[f]\left(z_{1}, z_{2}\right)=\frac{1}{\gamma_{\sigma} \gamma_{\tau}} \sum_{\mu=1}^{k} \sum_{\nu=1}^{l}(-1)^{\mu+\nu}\left(\begin{array}{c}
k \\
m
\end{array}\right)\left(\begin{array}{l}
l \\
\nu
\end{array}\right) \frac{1}{\mu \nu} S_{\mu, \nu}\left(z_{1}, z_{2}\right) .
$$

Therefore, the uniform relation (14) is established. Applying the Lebesgue dominated convergence theorem, it can easily be proved that the function $J_{\sigma, \tau}[f]$ is continuous at every point belonging to the space of pairs of complex numbers.

The (Lebesgue) integrals

$$
\int_{-n}^{n} \int_{-n}^{n} f(u, v) g_{\sigma}\left(\frac{z_{1}-u}{\mu}\right) g_{\tau}\left(\frac{z_{2}-v}{\nu}\right) d u d v \quad(\mu, \nu, n \in N)
$$

define some entire functions $F_{\mu, \nu, n}$ of two complex variables $z_{1}, z_{1}$, because they have the partial derivatives

$$
\frac{\partial F_{\mu, \nu, n}}{\partial z_{1}}\left(z_{1}, z_{2}\right)=\frac{1}{\mu} \int_{-n}^{n} \int_{-n}^{n} f(u, v) g_{\sigma}^{\prime}\left(\frac{z_{1}-u}{\mu}\right) g_{\tau}\left(\frac{z_{2}-v}{\nu}\right) d u d v
$$

and

$$
\frac{\partial F_{\mu, \nu, n}}{\partial z_{2}}\left(z_{1}, z_{2}\right)=\frac{1}{\nu} \int_{-n}^{n} \int_{-n}^{n} f(u, v) g_{\sigma}\left(\frac{z_{1}-u}{\mu}\right) g_{\tau}^{\prime}\left(\frac{z_{2}-v}{\nu}\right) d u d v
$$


An easy calculation shows that $\lim _{n \rightarrow \infty} F_{\mu, \nu, n}\left(z_{1}, z_{2}\right)=S_{\mu, \nu}\left(z_{1}, z_{2}\right)$ uniformly in $z_{1}, z_{2}$ belonging to two arbitrary bounded sets of complex numbers. Hence the well-known Weierstrass theorem ensures that all $S_{\mu, \nu}$ are entire functions of two variables. Consequently, $J_{\sigma, \tau}[f]$ is a bivariate entire function for every fixed pair $(\sigma, \tau)$ of positive numbers. Obviously, in view of $(14), J_{\sigma, \tau}[f](\cdot, \cdot) \in E_{\sigma, \tau}$.

Proposition 5. The singular integral (12), with a positive parameter $\sigma$, has the following basic properties:

(i) $J_{\sigma}^{1}[f](\cdot, v) \in E_{\sigma}$ for almost every $v \in R$,

(ii) $J_{\sigma}^{1}[f]\left(z_{1}, \cdot\right) \in L_{l o c}(R)$ for every complex $z_{1}$,

(iii) $J_{\sigma}^{1}[f](\cdot, \cdot) \in L_{l o c}\left(R^{2}\right)$.

Proof of (i). By Fubini's theorem, condition (10) implies

$$
\left|\int_{-\infty}^{\infty} \frac{f(u, v)}{1+u^{2 r}} d u\right|<\infty \text { and } \int_{-\infty}^{\infty} \frac{|f(u, v)|}{1+u^{2 r}} d u<\infty
$$

for almost every $v \in R$. Hence, for these $v$ and $z_{1}=x_{1}+i y_{1}$,

$$
\varphi_{\mu}\left(z_{1}, v\right) \equiv \int_{-\infty}^{\infty}\left|f(u, v) g_{\sigma}\left(\frac{z_{1}-u}{\mu}\right)\right| d u<\infty \quad(\mu=1, \ldots, k)
$$

and

$$
\begin{gathered}
Y\left(z_{1}, v\right) \equiv \int_{R} f(u, v) G_{\sigma, k}\left(z_{1}-u\right) d u= \\
=\sum_{\mu=1}^{k}(-1)^{\mu} \frac{1}{\mu}\left(\begin{array}{l}
k \\
\mu
\end{array}\right) \int_{-\infty}^{\infty} f(u, v) g_{\sigma}\left(\frac{z_{1}-u}{\mu}\right) d u
\end{gathered}
$$

Writing

$$
\varphi_{\mu}\left(z_{1}, v\right)=\left(\int_{x_{1}-1}^{x_{1}+1}+\int_{x_{1}+1}^{\infty}+\int_{-\infty}^{x_{1}-1}\right)\left|f(u, v) g_{\sigma}\left(\frac{z_{1}-u}{\mu}\right)\right| d u
$$

and proceeding as in the proof of Proposition 4, we obtain

$$
\varphi_{\mu}\left(z_{1}, v\right)=O\left(\left(1+x_{1}^{2 r}\right) e^{(\sigma / \mu)\left|y_{1}\right|} \int_{R}|f(u, v)|\left(1+u^{2 r}\right)^{-1} d u\right)
$$

uniformly in $x_{1}, y_{1}, v$. This immediately implies the $O$-relation for $Y\left(z_{1}, v\right)$, in which the right side is as in (16) with $\mu=1$.

Further, $Y(\cdot, v)$ are entire functions, by the Weierstrass theorem. Consequently, $Y(\cdot, v) \in E_{\sigma}$ for almost every $v$. Thus the assertion (i) is obtained. 
Proof of (ii). Given $\mu, n \in N$ and any complex number $z_{1}=x_{1}+i y_{1}$, we have

$$
\begin{gathered}
\int_{-\infty}^{\infty} \int_{-n}^{n}\left|f(u, v) g_{\sigma}\left(\frac{z_{1}-u}{\mu}\right)\right| d u d v \leq \\
\leq \int_{x_{1}-1}^{x_{1}+1} \int_{-n}^{n}|f(u, v)|\left(\frac{\sigma}{2 r}\right)^{2 r} e^{\sigma\left|y_{1}\right| / \mu} d u d v+ \\
+\left(\int_{-\infty}^{x_{1}-1} \int_{-n}^{n}+\int_{x_{1}+1}^{\infty} \int_{-n}^{n}\right)|f(u, v)|\left(\frac{\mu}{x_{1}-u}\right)^{2 r} e^{\sigma\left|y_{1}\right| / \mu} d u d v<\infty .
\end{gathered}
$$

Applying Fubini's theorem we conclude that $Y\left(z_{1}, \cdot\right) \in L_{l o c}(R)$, which implies (ii).

Proof of (iii). Let $\mu, n \in N, x_{1} \in R$. By Tonelli's theorem,

$$
\begin{aligned}
& \int_{-\infty}^{\infty} \int_{-n}^{n} \int_{-n}^{n}\left|f(u, v) g_{\sigma}\left(\frac{x_{1}-u}{\mu}\right)\right| d u d v d x_{1}= \\
= & \int_{-n}^{n}\left\{\int_{-\infty}^{\infty} \int_{-n}^{n}|f(u, v)| g_{\sigma}\left(\frac{x_{1}-u}{\mu}\right) d u d v\right\} d x_{1} .
\end{aligned}
$$

The inner double integral of the right-hand side of (17) does not exceed

$$
\begin{gathered}
\int_{x_{1}-1}^{x_{1}+1} \int_{-n}^{n}|f(u, v)|\left(\frac{\sigma}{2 r}\right)^{2 r} d u d v+ \\
+\left(\int_{-\infty}^{x_{1}-1} \int_{-n}^{n}+\int_{x_{1}+1}^{\infty} \int_{-n}^{n}\right)|f(u, v)|\left(\frac{\mu}{x_{1}-u}\right)^{2 r} d u d v \leq \\
\left.\leq\left(\frac{\sigma}{2 r}\right)^{2 r} \int_{-n-1}^{n+1} \int_{-n}^{n}|f(u, v)| d u d v+2 \mu^{2 r}(1+n)^{2 r}\right)\left(1+n^{2 r}\right) I(f) .
\end{gathered}
$$

Thus the left-hand side of (17) is finite.

Now, the Fubini theorem ensures that $Y(\cdot, \cdot) \in L_{l o c}\left(R^{2}\right)$, and (iii) follows.

Analogous properties of the singular integral (13) can easily be formulated. They will also be used in the sequel.

4. Approximations by the sums of pseudoentire functions. Let $f$ and the operators $J_{\sigma, \tau}, J_{\sigma}^{1}, J_{\tau}^{2}$ be as in Section 3. Putting

$$
\begin{gathered}
\Phi\left(z_{1}, v\right):=-J_{\sigma, \tau}[f]\left(z_{1}, v\right)-J_{\sigma}^{1}[f]\left(z_{1}, v\right), \\
\Psi\left(u, z_{2}\right):=J_{\tau}^{2}[f]\left(u, z_{2}\right),
\end{gathered}
$$

where $z_{1}=x_{1}+i y_{1}, z_{2}=x_{2}+i y_{2}\left(x_{j}, y_{j} \in R\right)$, and $u, v \in R$, it can easily be observed (see Propositions 4,5) that $\Phi(\cdot, \cdot) \in W_{\sigma}^{1}, \Psi(\cdot, \cdot) \in W_{\tau}^{2}$.

Introduce the approximant $Q_{\sigma, \tau}[f]\left(x_{1}, x_{2}\right):=\Phi\left(x_{1}, x_{2}\right)+\Psi\left(x_{1}, x_{2}\right)$, which is defined almost everywhere on $R^{2}$ and $Q_{\sigma, \tau}[f] \in L_{l o c}\left(R^{2}\right)$. Assuming that 
$k, l \leq 2 r-2(k, l, r \in N)$ and $1 \leq p \leq \infty$, we will present some Jackson type estimates.

In discussions below, the symbols $C_{j}(q, \ldots)$ will mean positive constants depending on the indicated parameters $q, \ldots$, only.

Theorem 1. Under the restriction $0<\sigma, \tau<\infty$, we have

$$
\left\|f-Q_{\sigma, \tau}[f]\right\|_{p} \leq 2^{k+l} C_{1}(r) \omega_{k, l}(1 / \sigma, 1 / \tau ; f)_{p} .
$$

Proof. Take into account the real numbers $x_{1}, x_{2}$ for which $J_{\sigma}^{1}[f]\left(\cdot, x_{2}\right) \in E_{\sigma}$, $J_{\tau}^{2}[f]\left(x_{1}, \cdot\right) \in E_{\tau}$. In this case,

$$
\begin{gathered}
f\left(x_{1}, x_{2}\right)-Q_{\sigma, \tau}[f]\left(x_{1}, x_{2}\right)= \\
=\frac{(-1)^{k+l}}{\gamma_{\sigma} \gamma_{\tau}} \iint_{R^{2}} \Delta_{s, t}^{k, l} f\left(x_{1}, x_{2}\right) g_{\sigma}(s) g_{\tau}(t) d s d t .
\end{gathered}
$$

Hence, by Minkowski's inequality and (5),

$$
\begin{gathered}
\gamma_{\sigma} \gamma_{\tau}\left\|f-Q_{\sigma, \tau}[f]\right\|_{p} \leq \\
\leq \omega_{k, l}(1 / \sigma, 1 / \tau ; f)_{p} \iint_{R^{2}}(\sigma|s|+1)^{k}(\tau|t|+1)^{l} g_{\sigma}(s) g_{\tau}(t) d s d t .
\end{gathered}
$$

Further,

$$
\begin{gathered}
\frac{1}{\gamma_{\sigma}} \int_{R}(\sigma|s|+1)^{k} g_{\sigma}(s) d s \leq \frac{2}{\gamma_{\sigma}}\left\{2^{k} \int_{0}^{1 / \sigma} g_{\sigma}(s) d s+\right. \\
\left.+2^{k} \sigma^{k} \int_{1 / \sigma}^{\infty} s^{k} g_{\sigma}(s) d s\right\} \leq 2^{k}\left\{1+\frac{2}{\gamma_{\sigma}} \sigma^{k} \int_{1 / \sigma}^{\infty} s^{k-2 r} d s\right\} \leq \\
\leq 2^{k}\left\{1+\frac{2}{\gamma_{\sigma}} \sigma^{2 r-1}\right\} .
\end{gathered}
$$

Observing that

$$
\gamma_{\sigma} \geq 2\left(\frac{\sigma}{2 r}\right)^{2 r-1} \int_{0}^{\pi / 2}\left(\frac{2}{\pi}\right)^{2 r} d v=2\left(\frac{\sigma}{\pi r}\right)^{2 r-1}
$$

we obtain

$$
\frac{1}{\gamma_{\sigma}} \int_{R}(\sigma|s|+1)^{k} g_{\sigma}(s) d s \leq 2^{k}\left\{1+(\pi r)^{2 r-1}\right\} .
$$

Thus

$$
\left\|f-Q_{\sigma, \tau}[f]\right\|_{p} \leq \omega_{k, l}(1 / \sigma, 1 / \tau ; f)_{p} \cdot 2^{k+l}\left\{1+(\pi r)^{2 r-1}\right\}^{2},
$$

and the proof is complete. 
Corollary 1. Let $f$ satisfy all conditions of Proposition 2, with some positive integers $m \leq k, n \leq l$. Then

$$
\left\|f-Q_{\sigma, \tau}[f]\right\|_{p} \leq 2^{k+l} C_{1}(r) \sigma^{-m} \tau^{-n} \omega_{k-m, l-n}\left(1 / \sigma, 1 / \tau ; f^{(m, n)}\right)_{p}
$$

This estimate is an immediate consequence of Theorem 1 and inequality (7).

Corollary 2. Consider the bivariate functions $f_{0}, f_{1}, \ldots, f_{\rho}$ defined in Proposition 3 , with $f_{0}(, 0), f_{0}(0, \cdot) \in L_{l o c}(R)$ and $f_{\rho-j}(\cdot, 0), f_{\rho-j}(0, \cdot) \in C(R)$ when $1 \leq j \leq \rho-1$. Suppose that for some non-negative numbers $a, b<$ $2 r-\rho-1$ and for $j=1, \ldots, \rho$ the relations

$$
\begin{gathered}
f_{\rho}(x, y)=O\left(\left(1+|x|^{a}\right)\left(1+|y|^{b}\right)\right), \\
\left.f_{\rho-j}(x, 0)=O\left(1+|x|^{a+j}\right), \quad f_{\rho-j}(0, y)=O\left(1+|y|^{b+j}\right)\right)
\end{gathered}
$$

hold uniformly in $x, y \in R$. Then if $k=l \geq \rho$, the function $f=f_{0}$ and its approximant $Q_{\sigma, \tau}[f]$ have weak derivatives of order $\rho-1$ everywhere on $R^{2}$ and

$$
\left\|f^{(\mu)}-Q_{\sigma, \tau}^{(\mu)}[f]\right\|_{p} \leq C_{2}(k, r)(\sigma \tau)^{\mu-\rho} \omega_{k-\rho+\mu, k-\rho+\mu}\left(\frac{1}{\sigma}, \frac{1}{\tau} ; f_{\rho}\right)_{p}
$$

for $\mu=0, \ldots, \rho-1$. Under the additional assumption $f_{\rho} \in C\left(R^{2}\right)$, also the derivatives $f^{(\rho)}$ and $Q_{\sigma, \tau}^{(\rho)}[f]$ exist on $R^{2}$ and the last inequality remains valid for $\mu=\rho$.

Indeed, an easy calculation shows that

$$
f^{(\mu)}(x, y)=f_{\mu}(x, y) \quad \text { and } \quad Q_{\sigma, \tau}^{(\mu)}[f](x, y)=Q_{\sigma, \tau}\left[f_{\mu}\right](x, y)
$$

for all $(x, y) \in R^{2}$, whenever $0 \leq \mu \leq \rho-1$ or $\mu=\rho$ and $f_{\rho} \in C\left(R^{2}\right)$. By Theorem 1 and a suitable estimate analogous to (9), we obtain

$$
\begin{gathered}
\left\|f_{\mu}-Q_{\sigma, \tau}[f]\right\|_{p} \leq C_{2}(k, r) \omega_{k, k}\left(1 / \sigma, 1 / \tau ; f_{\mu}\right)_{p} \leq \\
\leq C_{2}(k, r)(\sigma \tau)^{\mu-\rho} \omega_{k-\rho+\mu, k-\rho+\mu}\left(1 / \sigma, 1 / \tau ; f_{\rho}\right)_{p} \text { if } 0 \leq \mu \leq \rho .
\end{gathered}
$$

The conclusion is now evident.

Theorem 2. Let $f \in H_{\varphi, \psi, p}^{(k, l)}$, where $\varphi, \psi$ are as in Section 1. Denote by $\alpha$ and $\beta$ two non-negative numbers such that $t^{-\alpha} \varphi(t), t^{-\beta} \psi(t)$ are nondecreasing on $(0,1]$. Then, for $\sigma, \tau \geq 1$,

$$
\left\|f-Q_{\sigma, \tau}[f]\right\|_{\alpha, \beta, p}^{(k, l)} \leq C_{3}(k, l, r)\|f\|_{\varphi, \psi, p}^{(k, l)}\left\{\sigma^{\alpha} \varphi(1 / \sigma)+\tau^{\beta} \psi(1 / \tau)\right\} .
$$


Proof. Write $D_{\sigma, \tau}(x, y):=f(x, y)-Q_{\sigma, \tau}[f](x, y) \quad(x, y \in R)$ In view of (18),

$$
\begin{gathered}
\Delta_{\lambda, \eta}^{k, l} D_{\sigma, \tau}(x, y)=\frac{1}{\gamma_{\sigma} \gamma_{\tau}} \times \\
\times \iint_{R^{2}} g_{\sigma}(s) g_{\tau}(t) \sum_{\mu=0}^{k} \sum_{\nu=0}^{l}(-1)^{\mu+\nu}\left(\begin{array}{l}
k \\
\mu
\end{array}\right)\left(\begin{array}{l}
l \\
\nu
\end{array}\right) \Delta_{s, t}^{k, l} f(x+\mu \lambda, y+\nu \eta) d s d t
\end{gathered}
$$

for all $\lambda, \eta \in R$ and almost all $(x, y) \in R^{2}$; whence, by Minkowski's inequality,

$$
\left\|\Delta_{\lambda, \eta}^{k, l} D_{\sigma, \tau}\right\| \leq \frac{2^{k+l}}{\gamma_{\sigma} \gamma_{\tau}} \iint_{R^{2}} g_{\sigma}(s) g_{\tau}(t)\left\|\Delta_{s, t}^{k, l} f\right\|_{p} d s d t .
$$

Proceeding now as in the proof of Theorem 1, we obtain

$$
\left\|\Delta_{\lambda, \eta}^{k, l} D_{\sigma, \tau}\right\|_{p} \leq 4^{k+l} C_{1}(r)\|f\|_{\varphi, \psi, p}^{(k, l)} \varphi(1 / \sigma) \psi(1 / \tau) .
$$

On the other hand, equality (18) leads to

$$
\begin{gathered}
\Delta_{\lambda, \eta}^{k, l} D_{\sigma, \tau}(x, y)=\frac{1}{\gamma_{\sigma} \gamma_{\tau}} \times \\
\times \iint_{R^{2}} g_{\sigma}(s) g_{\tau}(t) \sum_{\mu=0}^{k} \sum_{\nu=0}^{l}(-1)^{\mu+\nu}\left(\begin{array}{l}
k \\
\mu
\end{array}\right)\left(\begin{array}{l}
l \\
\nu
\end{array}\right) \Delta_{\lambda, \eta}^{k, l} f(x+\mu s, y+\nu t) d s d t
\end{gathered}
$$

for almost all $(x, y) \in R^{2}$, which implies

$$
\left\|\Delta_{\lambda, \eta}^{k, l} D_{\sigma, \tau}\right\|_{p} \leq 2^{k+l}\|f\|_{\varphi, \psi, p}^{(k, l)} \varphi(\lambda) \psi(\eta) \text { when } \lambda, \eta \in(0,1] .
$$

Next, $\left\|D_{\sigma, \tau}\right\|_{\alpha, \beta, p}^{(k, l)}=\sup \{\Omega(\lambda, \eta): 0<\lambda, \eta \leq 1\}$, where $\Omega(\lambda, \eta)=$ $\lambda^{-\alpha} \eta^{-\beta}\left\|\Delta_{\lambda, \eta}^{k, l} D_{\sigma, \tau}\right\|_{p}$. If $1 / \sigma \leq \lambda \leq 1$ and $1 / \tau \leq \eta \leq 1$ then, by (19), $\Omega(\lambda, \eta) \leq 4^{k+l} C_{1}(r)\|f\|_{\varphi, \psi, p}^{(k, l)} \sigma^{\alpha} \varphi(1 / \sigma) \tau^{\beta} \psi(1 / \tau)$. From (20) it follows that

$$
\begin{gathered}
\Omega(\lambda, \eta) \leq 2^{k+l}\|f\|_{\varphi, \psi, p}^{(k, l)} \times \\
\times \begin{cases}\sigma^{\alpha} \varphi(1 / \sigma) \tau^{\beta} \psi(1 / \tau) & \text { if } 0<\lambda \leq 1 / \sigma, 0<\eta \leq 1 / \tau, \\
\sigma^{\alpha} \varphi(1 / \sigma) & \text { if } 0<\lambda \leq 1 / \sigma, 1 / \tau \leq \eta \leq 1, \\
\tau^{\beta} \psi(1 / \tau) & \text { if } 1 / \sigma \leq \lambda \leq 1,0<\eta \leq 1 / \tau .\end{cases}
\end{gathered}
$$

Hence

$$
\begin{gathered}
\left\|D_{\sigma, \tau}\right\|_{\alpha, \beta, p}^{(k, l)} \leq 2^{k+l}\|f\|_{\varphi, \psi, p}^{(k, l)}\left\{2^{k+l} C_{1}(r) \sigma^{\alpha} \varphi(1 / \sigma) \tau^{\beta} \psi(1 / \tau)+\right. \\
\left.+\sigma^{\alpha} \varphi(1 / \sigma) \tau^{\beta} \psi(1 / \tau)+\sigma^{\alpha} \varphi(1 / \sigma)+\tau^{\beta} \psi(1 / \tau)\right\},
\end{gathered}
$$

and the proof is completed (cf. [4], Theorem 2). 
Corollary 3. If $\varphi(\delta)=\delta^{\alpha^{\prime}}, \psi(\delta)=\delta^{\beta^{\prime}}$ for all $\delta \in(0,1]$ then, in the case $\alpha^{\prime} \geq \alpha \geq 0$ and $\beta^{\prime} \geq \beta \geq 0,\left\|f-Q_{\sigma, \tau}[f]\right\|_{\alpha, \beta, p}^{(k, l)}=O\left(\sigma^{\alpha-\alpha^{\prime}}+\tau^{\beta-\beta^{\prime}}\right)$ uniformly in $\sigma, \tau \geq 1$.

\section{REFERENCES}

1. S.N.Bernstein, Collected Papers, vol. II. (Russian) Izdat. Akad. Nauk SSSR, Moscow, 1954.

2. Yu.A.Brudnyi, Approximation of functions of $n$ variables by quasipolynomials. (Russian) Izv. Akad. Nauk SSSR, Ser. Mat. 34(1970), 564-583.

3. H.Gonska and K.Jetter, Jackson-type theorems on approximation by trigonometric and algebraic pseudopolynomials. J. Approx. Theory 48(1986), 396-406.

4. R.Taberski, Approximation properties of the integral Bernstein operators and their derivatives in some classes of locally integrable functions. Funct. Approx. Comment. Math. 21 (in press).

5. A.F.Timan, Theory of Approximation of Functions of a Real Variable. (Russian) Gosudarstv. Izdat. Fiz.-Mat. Lit., Moscow, 1960.

(Received 20. XI. 1992)

Author's address:

Institute of Mathematics

Adam Mickiewicz University

Matejki 48/49

60-769 Poznań, Poland 